\title{
Performance Modeling Analysis of D-MSMR-CARQ with Relay Selection in Wireless Sensor Networks
}

\author{
Yongqiang Zhou (D), Huan Qian, Qihao Wang, and Suoping Li \\ School of Science, Lanzhou University of Technology, Lanzhou 730050, China \\ Correspondence should be addressed to Yongqiang Zhou; zhoupaper@126.com
}

Received 21 February 2021; Revised 3 June 2021; Accepted 25 June 2021; Published 8 July 2021

Academic Editor: Leonardo Mostarda

Copyright (C) 2021 Yongqiang Zhou et al. This is an open access article distributed under the Creative Commons Attribution License, which permits unrestricted use, distribution, and reproduction in any medium, provided the original work is properly cited.

\begin{abstract}
Reliable and efficient real-time transmission is an important and challenging issue for wireless sensor networks (WSNs). Truncated retransmission times and relay selection can effectively reduce transmission delay and improve system throughput. A new direct multisource multirelay cooperative automatic repeat request (D-MSMR-CARQ) protocol based on truncation with two relay selection methods in WSNs is analytically analyzed in this paper. Firstly, based on two different relay selection methods under the maximum ratio combining (MRC), the discrete time Markov chain (DTMC) model of D-MSMR-CARQ protocol and state space is established. Secondly, for each D-MSMR-CARQ protocol based on different relay selection method, we obtain the closed-form expressions of the system average transmission delay and the expressions of the system throughput through state transition probabilities. Finally, numerical results reveal that the first relay selection method outperforms the second relay selection method on the average transmission delay performance for the proposed protocol. More specifically, the delay performance of the proposed protocol can be improved by $13 \%$ compared with the nondirect-link protocol when the channel environment is the same; the proposed protocol improves the throughput performance by $47 \%$ compared with the nondirect protocol when the channel environment is harsh under the same simulation parameters. Furthermore, the optimal number of source nodes and relay nodes is determined.
\end{abstract}

\section{Introduction}

With the progress of wireless mobile communication technology and Internet of things (IoT), WSNs have broad application prospects in many fields such as environmental monitoring [1-3], smart agriculture [4], smart city [5, 6], and smart grid $[7,8]$. However, WSNs face many challenges in system delay, throughput, and energy consumption. Therefore, it is a very practical problem to solve the reliability, antifading ability, effectiveness, and energy limitation of sensor nodes for WSNs.

As the major error control technology, automatic repeat request (ARQ) is favored by WSNs for its low complexity, severe energy constraints, reliable data transmission, and lower power communication requirements. Sarvi et al. [9] introduced an improved hybrid FEC/ARQ method for multimedia streaming over WSNs and derived the stationary probabilities and the delay performance. Li et al. [10] designed five broadcast-based ARQ protocols in multihop WSNs and analyzed the maximum number of retransmissions and the minimum energy consumption. The impacts of error control schemes (ARQ, FEC, and hybrid ARQ) on the network lifetime of WSNs are investigated in [11]. However, the network throughput of ARQ protocols in WSNs under stable state has not been studied.

Multiple input multiple output (MIMO) technology can improve the antifading ability by increasing the spatial diversity gain. Dey et al. [12] reported an indoor-outdoor measurement campaign in virtual MIMO WSNs and analyzed the collected data and the average MIMO capacity. Zhang et al. [13] analyzed the security performance of multigroup multicast centralized large-scale MIMO system under active attack. However, for WSNs composed of sensor nodes that are not easy to harvest energy, it is impractical to 
install multiple antennas due to the size and energy constraints of sensor nodes. Cooperative communication (CC) technology uses the broadcast characteristics of the channel, the source node broadcasts data packets to the relay node and the destination node at the same time, and the relay node cooperates with the source node to transmit data packets to form a virtual antenna array, to achieve the effect of MIMO. Therefore, a new CMIMO framework was proposed in [14] to save energy, which combined CC technology with MIMO technology and utilized cooperation between source node and relay node for long distance communication. Asheer and Kumar [15] verified that CMIMO can significantly improve the performance of WSNs and maintain the required quality of service by improving throughput, energy saving, and reliability. Furthermore, ARQ technology is combined with CC to form cooperative ARQ (CARQ) technology. CARQ not only ensures the high reliability of data transmission but also increases the spatial diversity gain, so it becomes the most suitable solution for WSNs. Wang et al. [16] suggested a single-source two-relay nodes selection CARQ protocol and built a discrete time Markov chain (DTMC) model to calculate the energy efficiency and throughput gain in WSNs. Tseng and Wu [17] proposed two types of selective and opportunistic AF relaying mechanisms for CARQ protocol; the closed-form expression of outage probability was derived. However, the average packet transmission delay of CARQ protocol in WSNs was not discussed.

Energy consumption is the core problem of WSNs; scholars have found that relay selection technology can effectively reduce energy consumption and improve energy efficiency. Torabi et al. [18] investigated the amplify-andforward (AF) cooperative diversity system with best relay selection and derived the end-to-end signal-to-noise ratio (SNR) under the assumption of MRC and obtained the closed-form expressions of outage probability and average symbol error rate (SER). Arro-Valles et al. [19] proposed a sparsity-aware consistent sensor-relay-and-link selection algorithm based on optimal sparse solutions and convex relaxation technique. Bissias et al. [20] employed the best single-relay selection algorithm to study the outage probability and the average bit error rate (BER) for dual-hop relay system. Zou et al. [21] adopted an optimal three-stage relay selection scheme to derive the joint probability density function (PDF), the signal to interference and noise ratio (SINR), the optimal relay selection criterion, and the outage probability. Rahman et al. [22] considered the K-means estimation approach in 2-tier WSNs and selected the closest relay node to the base station to reduce energy consumption; the delay and throughput performance were not analyzed. However, if there is abnormal cluster, the mean deviation will be serious.

CC technology forwards the data packet from the source node through the relay node; thus, the communication link of users can be strengthened and CARQ technology can simultaneously reduce the packet error rate and improve the system throughput. Therefore, Zhou et al. [23] proposed a multisource single-relay CARQ protocol, but the single relay cannot meet the signal coverage required by current users
[24, 25]. Li et al. [26] studied the single-source multirelay CARQ protocol and analyzed its throughput and delay performance by establishing the DTMC model. On this basis, combined with the abovementioned work, a multisource multirelay CARQ without direct-link protocol was proposed [27, 28]. However, due to the different channel environment of each link in the system, the improvement of performance is not obvious for the medium and long distance multisource multirelay communication system.

In this paper, we consider a basic medium and long distance cellular communication scenario, in which the source node can communicate with the destination node directly. In order to improve the reliability of data transmission, multiple relays are used for cooperative communication. In addition, unlike the case in [28] in which the direct-link was not considered, the direct-link provides additional performance improvement when the channel environment is harsh. Therefore, we present a new D-MSMR-CARQ protocol which is suitable for WSNs. The main contributions of this paper can be summarized as follows:

(1) A new direct multisource multirelay protocol based on truncated CARQ (D-MSMR-CARQ) is proposed for the communication system. The D-MSMRCARQ protocol includes the coexistence of one-hop direct transmission and dual-hop transmission. Compared with traditional CC, the protocol achieves significant gains in delay and throughput performance.

(2) Based on two different relay selection schemes, we obtain the best relay node and summarize the packet transmission process of the D-MSMR-CARQ protocol into two DTMC models and derive the state space, respectively.

(3) For each D-MSMR-CARQ protocol based on different relay selection methods, we derive the closedform expressions of the average transmission delay by using the average PER and the expressions of the throughput by the state transition probabilities, respectively.

(4) By properly setting the system parameters, numerical results show that we conclude several guidelines for the D-MSMR-CARQ protocol: firstly, there exists the optimal number of relays and sources deployed in the system when maximizing the throughput and minimizing the transmission delay. Secondly, for the proposed protocols under two relay selection methods, the protocol based on the first relay selection method has better delay and throughput performance. Thirdly, direct-link transmission cannot be ignored when the channel environment is harsh. The reason is that for the MSMR-CARQ with nondirect-link protocol, our proposed protocol improves the delay performance by $13 \%$; moreover, our proposed protocol improves the throughput performance by $47 \%$ when the PER from the source node to the destination node is 0.8 . 
The rest of this paper is organized as follows. The system DTMC models and the state spaces are described in Section 2. In Section 3, we derive the closed-form expressions for system average transmission delay of the D-MSMR-CARQ protocol. The state transition probabilities and the network throughput performance analysis of the proposed are carried out in Section 4. The numerical results are provided in Section 5. Finally, we conclude the paper in Section 6.

\section{System Model and Analysis}

Supposing that each source node sends the same data packet, the data packet obeys the Bernoulli process at the arrival rate $\lambda(0<\lambda<1)$, the data transmission follows the first come first service (FCFS) rule, and the packet service delay follows the independent and identically distributed (i.i.d.) arrival process. We consider a network, as shown in Figure 1, which consists of $M$ source nodes $S_{i}(i=1,2, \ldots, M)$ and $N$ relay nodes $R_{j}(j=1,2, \ldots, N)$, and a destination node $D$, from $S_{i}$ to $D$, adopts one-hop direct-link and dual-hop mechanisms, and $S_{i}$ broadcasts the same data packet to $R_{j}$ and $D$ at the same time. In practice, $M$ source nodes can represent broadcast towers of the next generation broadcasting wireless network, which can provide the same signals to mobile phones, TV, public $\mathrm{Wi}-\mathrm{Fi}$, and so on; $N$ relay nodes and destination node $D$ have different quality of service. By combining direct-link transmission with CC technique, the data packet can be transmitted more reliably. The purpose is that when the direct-link transmission fails, the CC technique is started to improve the reliability of the system. In addition, to improve the data transmission efficiency of WSNs and increase the throughput of the system, this paper uses two relay selection methods under the MRC method.

(a) First is adopting the first relay selection method, which is to select a corresponding best relay for each source node $S_{i}(i=1,2, \ldots, M)$. At this point, the system model can be simplified to Figure 2 .

According to [18], by using the MRC method, we can select the best relay $R_{b_{i}}$ for each source node in the first relay selection method as

$R_{b_{i}}=\arg \max _{j=1,2, \ldots, N} \min \left(\gamma_{S_{i} R_{j}}, \gamma_{R_{j} D}\right), \quad i=1,2, \ldots, M$,

where $\quad \gamma_{S_{i} R_{j}}=\left|h_{S_{i} R_{j}}\right|^{2} E_{S_{i}} / N_{0}, \quad \gamma_{R_{j} D}=\left|h_{R_{j} D}\right|^{2} E_{S_{i}} / N_{0}$ denote the instantaneous SNR of $S_{i}-R_{j}$ and $R_{j}-D$, respectively, and $E_{S_{i}}$ represents the average energy of each symbol, $\min \left(\gamma_{S_{i} R_{j}}, \gamma_{R_{j} D}\right) \geq\left(\gamma_{S_{i} R_{j}} \gamma_{R_{j} D} / 1+\gamma_{S_{i} R_{j}}{ }^{+}\right.$ $\left.\gamma_{R_{j} D}\right)$. Let $h_{x y}$ denote the channel gain between node $x$ and node $y, n_{x y}$ represents complex additive white Gaussian noise (CAWGN), $n_{x y}$ can be expressed as $n_{x y} \sim C N\left(0, N_{0}\right)$, where $x=\left\{S_{i}, R_{j}\right\}, y=\left\{R_{j}, D\right\}$, and $N_{0}$ is the noise variance.

For each group transmission system in the first relay selection method, the packet transmission process of the D-MSMR-CARQ protocol can be reduced to a 3state DTMC model, as shown in Figure 3, where

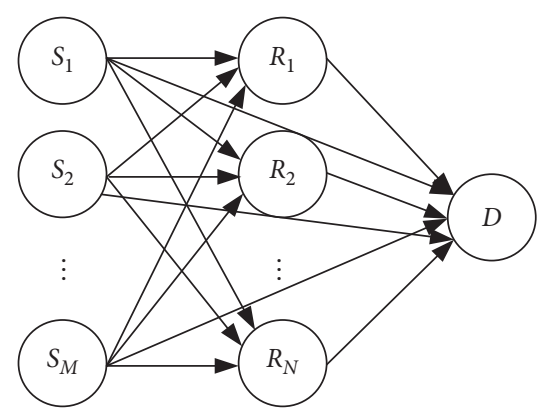

FIgURE 1: D-MSMR-CARQ system model.

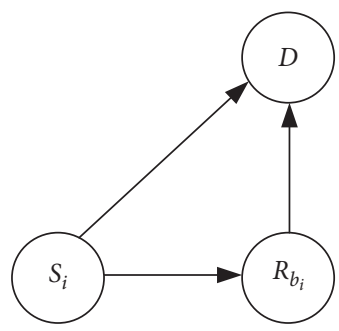

FIGURE 2: D-MSMR-CARQ system model in the first relay selection method.

$p_{s t}\left(s, t=0^{\prime}, b_{i}^{\prime},(N+1)^{\prime}\right)$ is the transition probability from node $s$ to node $t$ in the state space $\Omega^{\prime}=\left\{0^{\prime}, b_{i}^{\prime},(N+1)^{\prime}\right\}$.

The state space can be explained as follows:

State $0^{\prime}$ : the destination node $D$ and all relay nodes $R_{j}(j=1,2, \ldots, N)$ feed back nonacknowledgement (NACK) signals to the source node $S_{i}(i=1,2, \ldots$, $M)$, then the source node will retransmit the packet in the next time slot;

State $b_{i}^{\prime}$ : the destination node $D$ feeds back a NACK signal to the source node $S_{i}(i=1,2, \ldots, M)$, and the best relay node $R_{b_{i}}$ feeds back an acknowledgement (ACK) signal to the source node $S_{i}$; at this time, the best relay node $R_{b_{i}}$ will replace the source node $S_{i}$ to forward the packet in the next slot. However, the destination node $D$ feeds back a NACK signal to $R_{b_{i}}$, indicating that $D$ did not receive the data packet forwarded by $R_{b_{i}}$ correctly. Then, $R_{b_{i}}$ will retransmit the packet to $D$ in the next time slot;

State $(N+1)^{\prime}$ : the destination node $D$ feeds back an ACK signal to the source node $S_{i}$, then the source node $S_{i}$ will transmit a new data packet in the next time slot.

(b) Second is adopting the second relay selection method, in which all the source nodes use the MRC method to select a common best relay $R_{b}$ as

$$
R_{b}=\arg \max _{j=1,2, \ldots, N} \min _{i=1,2, \ldots, M}\left(\gamma_{S_{i} R_{j}}, \gamma_{R_{j} D}\right) .
$$

At this point, the system model can be simplified to Figure 4 .

The packet transmission process of the D-MSMRCARQ protocol in the second relay selection method 


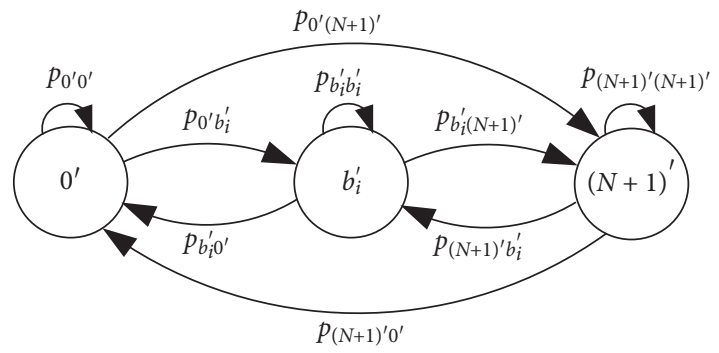

Figure 3: D-MSMR-CARQ state transition in the first relay selection method.

is similar to the DH-MSMR-CARQ protocol in the second relay selection method [28]. It can be reduced to a $2 M+4$ state DTMC model, as Figure 5 shows, where $p_{s t}\left(s, t=0^{\prime}, 1^{\prime}, \ldots,(2 M+3)^{\prime}\right)$ is the transition probability from node $s$ to node $t$ in the state space $\Omega^{\prime}=\left\{0^{\prime}, 1^{\prime}, \ldots,(2 M+3)^{\prime}\right\}$.

The state space can be explained as follows:

State $0^{\prime}$ : the destination node $D$ and the best relay $R_{b}$ feed back NACK signals to all source nodes $S_{i}(i=1,2, \ldots, M)$, indicating that neither $D$ nor $R_{b}$ received the data packet from $S_{i}$ correctly. Then, the packet will be retransmitted by $S_{i}$ in the next time slot;

State $i^{\prime}\left(i^{\prime}=1,2, \ldots, M\right)$ : the destination node $D$ feeds back NACK signals to all source nodes $S_{i}(i=1,2, \ldots, M)$, then the best relay $R_{b}$ feeds back an ACK signal to a certain source node $S_{i^{\prime}}$ and NACK signals to the remaining source nodes $S_{j}\left(j=1,2, \ldots, i^{\prime}-1, i^{\prime}+1, \ldots, M\right)$, indicating that $R_{b}$ has received the data packet from $S_{i^{\prime}}$ correctly. At this time, $R_{b}$ will replace $S_{i^{\prime}}$ to forward the packet in the next slot. However, the destination node $D$ feeds back a NACK signal to $R_{b}$, indicating that $D$ did not receive the data packet forwarded by $R_{b}$ correctly. Then, $R_{b}$ will retransmit the packet to $D$ in the next slot;

State $(M+1)^{\prime}$ : the destination node $D$ feeds back NACK signals to all source nodes $S_{i}(i=1,2, \ldots, M)$, and the best relay $R_{b}$ feeds back ACK signals to two or more source nodes $S_{i^{\prime}}, S_{j^{\prime}}, \ldots$, indicating that $R_{b}$ has received the data packets from $S_{i^{\prime}}, S_{j^{\prime}}, \ldots$ correctly. At this time, $R_{b}$ combines the data from $S_{i^{\prime}}, S_{j^{\prime}}, \ldots$, and forwards the combined packet in the next time slot. However, the destination node $D$ feeds back a NACK signal to $R_{b}$, indicating that $D$ did not receive the data packet forwarded by $R_{b}$ correctly. Then, $R_{b}$ will retransmit the packet to $D$ in the next slot;

State $(M+k)^{\prime}(k=2,3, \ldots, M+1)$ : the destination node $D$ feeds back NACK signals to all source nodes $S_{i}(i=1,2, \ldots, M)$, and the best relay $R_{b}$ feeds back an ACK signal to a certain source node $S_{k-1}$ and

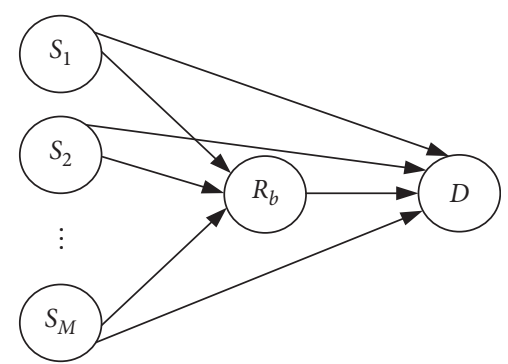

FIgURE 4: D-MSMR-CARQ system model in the second relay selection method.

NACK signals to the remaining source nodes $S_{j}(j=1,2, \ldots, k-2, k, \ldots, M+1)$, indicating that $R_{b}$ has received the data packet from $S_{k-1}$ correctly. At this time, $R_{b}$ will replace $S_{k-1}$ to forward the packet in the next slot. However, the destination node $D$ feeds back an ACK signal to $R_{b}$, indicating that $D$ has received the data packet forwarded by $R_{b}$ correctly. Then, the source node $S_{i}(i=1,2, \ldots, M)$ will transmit a new packet in the next time slot;

State $(2 M+2)^{\prime}$ : the destination node $D$ feeds back NACK signals to all source nodes $S_{i}(i=1,2, \ldots, M)$, and the best relay $R_{b}$ feeds back ACK signals to two or more source nodes $S_{i^{\prime}}, S_{j^{\prime}}, \cdots$. However, the destination node $D$ feeds back an ACK signal to $R_{b}$ .Then, the source node $S_{i}(i=1,2, \ldots, M)$ will transmit a new packet in the next time slot;

State $(2 M+3)^{\prime}$ : the destination node $D$ feeds back an ACK signal to at least one source node. At this time, regardless of whether the best relay $R_{b}$ feeds back an ACK signal or a NACK signal to the source node $S_{i}(i=1,2, \ldots, M)$, the source node $S_{i}(i=1,2, \ldots, M)$ will transmit a new packet in the next slot.

\section{Delay Performance Analysis}

According to Markov chain model and the analysis of state space in Section 2, we can get the delay performance of D-MSMR-CARQ protocol based on two relay selection methods.

(a) The average packet error rate (PER) of D-MSMRCARQ protocol in the first relay selection method is

$$
\operatorname{PER}_{\text {D-MSMR-CARQ }}=\prod_{i=1}^{M}\left[p_{S_{i} D} p_{S_{i} R_{b_{i}}}+p_{S_{i} D}\left(1-p_{S_{i} R_{b_{i}}}\right) p_{R_{b_{i}} D}\right] \text {, }
$$

where $p_{S_{i} R_{b_{i}}}, p_{R_{b_{i}} D}$ denote PER of $S_{i}-R_{b_{i}}$ and $R_{b_{i}}-D$, respectively.

Therefore, based on truncation, we can obtain the packet transmission delay of the D-MSMR-CARQ protocol in the first relay selection method as 


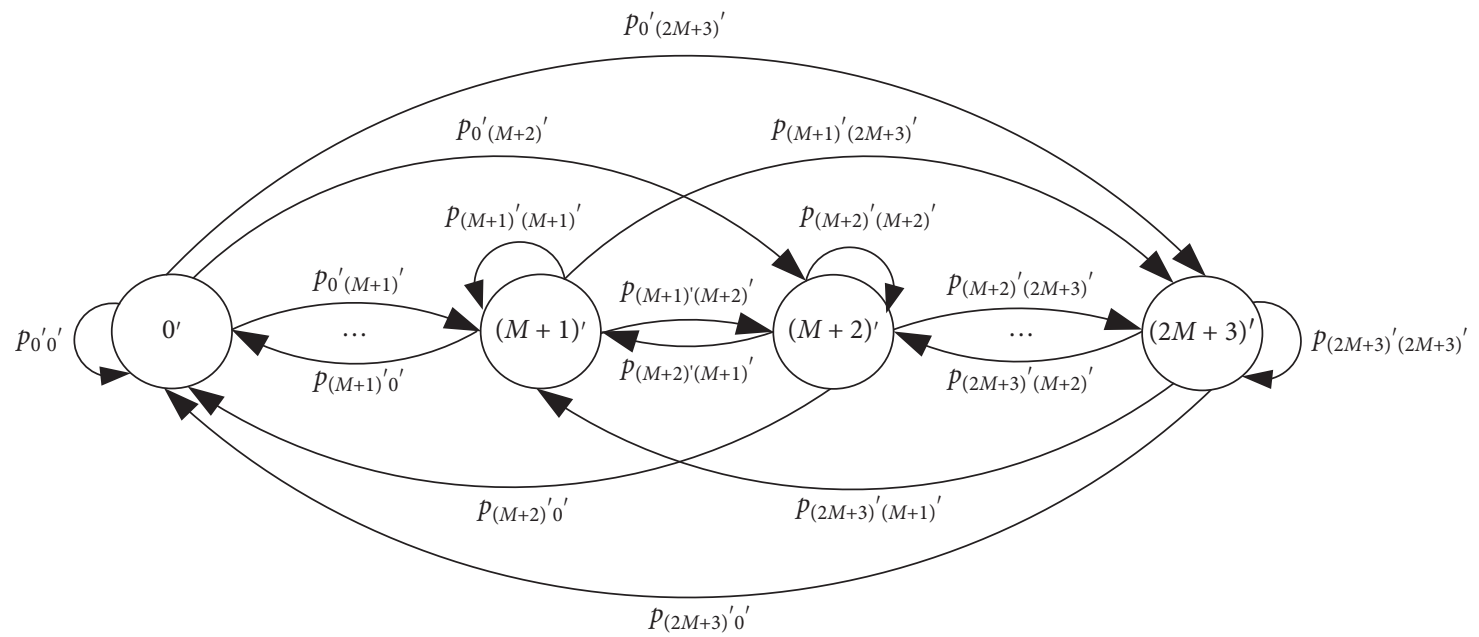

FiguRE 5: D-MSMR-CARQ state transition in the second relay selection method.

$$
S_{\text {D-MSMR-CARQ }}=t_{T} \frac{\left\{1-\left[\prod_{i=1}^{M}\left[p_{S_{i} D} p_{S_{i} R_{b_{i}}}+p_{S_{i} D}\left(1-p_{S_{i} R_{b_{i}}}\right) p_{R_{b_{i}} D}\right]\right]^{K}\right\}}{\left[1-\prod_{i=1}^{M}\left[p_{S_{i} D} p_{S_{i} R_{b_{i}}}+p_{S_{i} D}\left(1-p_{S_{i} R_{b_{i}}}\right) p_{R_{b_{i}} D}\right]\right]}+t_{p},
$$

where $K$ denotes the truncated times, $t_{T}$ is the transmission time of a packet, and $t_{p}$ is the propagation time of a packet.
Then, we can obtain the mathematical expectation of $S_{\text {D-MSMR-CARQ, }}$ which is defined as the average transmission delay of the D-MSMR-CARQ protocol in the first relay selection method as

$$
\begin{aligned}
E\left(S_{\mathrm{D}-\mathrm{MSMR}-\mathrm{CARQ}}\right) & =\frac{t_{T}}{\left(1-\mathrm{PER}_{\mathrm{D}-\mathrm{MSMR}-\mathrm{CARQ}}\right)}+t_{p} \\
& =\frac{t_{T}}{\left\{1-\prod_{i=1}^{M}\left[p_{S_{i} D} p_{S_{i} R_{b_{i}}}+p_{S_{i} D}\left(1-p_{S_{i} R_{b_{i}}}\right) p_{R_{b_{i}} D}\right]\right\}}+t_{p} .
\end{aligned}
$$

(b) Based on state space, we can derive the average PER of D-MSMR-CARQ protocol in the second relay selection method as

$$
\mathrm{PER}_{\mathrm{D}-\mathrm{MSMR}-\mathrm{CARQ}}^{\prime}=\prod_{i=1}^{M}\left[p_{S_{i} D} p_{S_{i} R_{b}}+p_{S_{i} D}\left(1-p_{S_{i} R_{b}}\right) p_{R_{b} D}\right]
$$

where $p_{S_{i} R_{b}}, p_{R_{b} D}, p_{S_{i} D}$ denote the PER of $S_{i}-R_{b}$, $R_{b}-D$, and $S_{i}-D$, respectively.

Therefore, based on truncation, we can obtain the packet transmission delay of D-MSMR-CARQ protocol in the second relay selection method as

$$
S_{\text {D-MSMR-CARQ }}^{\prime}=t_{T} \frac{\left\{1-\left[\prod_{i=1}^{M}\left[p_{S_{i} D} p_{S_{i} R_{b}}+p_{S_{i} D}\left(1-p_{S_{i} R_{b}}\right) p_{R_{b} D}\right]\right]^{K}\right\}}{\left[1-\prod_{i=1}^{M}\left[p_{S_{i} D} p_{S_{i} R_{b}}+p_{S_{i} D}\left(1-p_{S_{i} R_{b}}\right) p_{R_{b} D}\right]\right]}+t_{p} .
$$

Then, we can obtain the mathematical expectation of

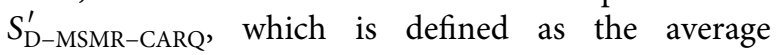

transmission delay of D-MSMR-CARQ protocol system in the second relay selection method as 


$$
\begin{aligned}
E\left(S_{\mathrm{D}-\mathrm{MSMR}-\mathrm{CARQ}}^{\prime}\right) & =\frac{t_{T}}{\left(1-\mathrm{PER}_{\mathrm{D}-\mathrm{MSMR}-\mathrm{CARQ}}^{\prime}\right)}+t_{p} \\
& =\frac{t_{T}}{\left\{1-\prod_{i=1}^{M}\left[p_{S_{i} D} p_{S_{i} R_{b}}+p_{S_{i} D}\left(1-p_{S_{i} R_{b}}\right) p_{R_{b} D}\right]\right\}}+t_{p} .
\end{aligned}
$$

\section{Throughput Performance Analysis}

(a) When the average number of packets reached within a packet transmission delay is $\rho=\lambda E\left(S_{\text {D-MSMR-CARQ }}\right)<1$, see $[23,24]$, the throughput of D-MSMR-CARQ protocol in the first relay selection method can be derived as

$$
T h_{\text {D-MSMR-CARQ }}=\sum_{i=1}^{M}\left\{\frac{1-p_{S_{i} D} \prod_{j=1}^{N} p_{S_{i} R_{j}}}{p_{S_{i} D} \prod_{j=1}^{N} p_{S_{i} R_{j}}}\left[1+\frac{1-p_{S_{i} D} \prod_{j=1}^{N} p_{S_{i} R_{j}}}{p_{S_{i} D} \prod_{j=1}^{N} p_{S_{i} R_{j}}}+\frac{1-\prod_{j=1}^{N} p_{S_{i} R_{j}}}{\left(1-p_{R}\right) \prod_{j=1}^{N} p_{S_{i} R_{j}}}\right]^{-1}\right\}
$$

where $p_{R}=\left(p_{R_{b_{i}}} p_{R_{b_{i}} D}+1-p_{R_{b_{i}}}\right)\left(p_{S_{i}} p_{S_{i} D}+1-p_{S_{i}}\right)$.

(b) From the system model based on the second relay selection method in Section 2 and its $2 M+4$ state space definition, we can see that $\Omega^{\prime}=\left\{0^{\prime}, 1^{\prime}, \ldots,(2 M+3)^{\prime}\right\}$ is a complete DTMC state space, and the DTMC model has a smooth transition probability. The state transition probabilities of the system are

$$
\left\{\begin{array}{l}
p_{0^{\prime} 0^{\prime}}=\prod_{i=1}^{M} p_{S_{i} D} p_{S_{i} R_{b}} ; \\
p_{0^{\prime} i^{\prime}}=\left(1-p_{S_{i}} R_{b}\right) p_{R_{b} D} \prod_{i=1}^{M} p_{S_{i} D} \prod_{j=1}^{i-1} p_{S_{j} R_{b}} \prod_{j=i+1}^{M} p_{S_{j} R_{b}}, \quad i=1,2, \ldots, M ; \\
p_{0^{\prime}(M+1)^{\prime}}=\prod_{i=1}^{M} p_{S_{i} D}\left[\prod_{i=1}^{2}\left(1-p_{S_{i} R_{b}}\right) \prod_{j=3}^{M} p_{S_{j} R_{b}}+\left(1-p_{S_{1} R_{b}}\right)\left(1-p_{S_{3} R_{b}}\right) p_{S_{2} R_{b}} \prod_{j=4}^{M} p_{S_{j} R_{b}}+\cdots+p_{S_{M} R_{b}} \prod_{i=1}^{M-1}\left(1-p_{S_{i} R_{b}}\right)+\prod_{i=1}^{M}\left(1-p_{S_{i} R_{b}}\right)\right] p_{R_{b} D} ; \\
p_{0^{\prime}(2 M+2)^{\prime}}=\prod_{i=1}^{M} p_{S_{i} D}\left[\prod_{i=1}^{2}\left(1-p_{S_{i} R_{b}}\right) \prod_{j=3}^{M} p_{S_{j} R_{b}}+\left(1-p_{S_{1} R_{b}}\right)\left(1-p_{S_{3} R_{b}}\right) p_{S_{2} R_{b}} \prod_{j=4}^{M} p_{S_{j} R_{b}}+\ldots+p_{S_{M} R_{b}} \prod_{i=1}^{M-1}\left(1-p_{S_{i} R_{b}}\right)+\prod_{i=1}^{M}\left(1-p_{S_{i} R_{b}}\right)\right]\left(1-p_{R_{b} D}\right) ; \\
p_{0^{\prime}(2 M+3)^{\prime}}=1-\prod_{i=1}^{M} p_{S_{i} D} ; \\
p_{i^{\prime} 0^{\prime}}=0, \quad i=1,2, \ldots, M+1 ; \\
p_{i^{\prime} j}=\left(p_{R_{b}} p_{R_{b} D}+1-p_{R_{b}}\right)\left(p_{S} \prod_{i=1}^{M} p_{S_{i} D}+1-p_{S}\right), \quad i, j=1,2, \ldots, M+1 ; \\
p_{i^{\prime}(M+k)^{\prime}}=\left[p_{R_{b}}\left(1-p_{R_{b} D}\right)\left(1-p_{S_{k-1} R_{b}}\right) \prod_{i=1}^{k-2} p_{S_{i} R_{b}} \prod_{i=k}^{M} p_{S_{i} R_{b}}\right]\left(p_{S} \prod_{i=1}^{M} p_{S_{i} D}+1-p_{S}\right), \quad i=1,2, \ldots, M+1, k=2,3, \ldots, M+1 ; \\
p_{i^{\prime}(2 M+2)^{\prime}}=\left(p_{S} \prod_{i=1}^{M} p_{S_{i} D}+1-p_{S}\right)\left[1-(M+1)\left(p_{R_{b}} p_{R_{b} D}+1-p_{R_{b}}\right)-\sum_{k=2}^{M+1}\left[p_{R_{b}}\left(1-p_{R_{b} D}\right)\left(1-p_{S_{k-1} R_{b}}\right) \prod_{i=1}^{k-2} p_{S_{i} R_{b}} \prod_{i=k}^{M} p_{S_{i} R_{b}}\right], \quad i=1,2, \ldots, M+1 ;\right. \\
p_{i^{\prime}(2 M+3)^{\prime}}=p_{S}\left(1-\prod_{i=1}^{M} p_{S_{i} D}\right), \quad i=1,2, \ldots, M+1 ; \\
p_{j^{\prime} k^{\prime}}=p_{0^{\prime} k^{\prime}}, \quad j=M+2, M+3, \ldots, 2 M+3, k=0,1, \ldots, 2 M+3 .
\end{array}\right.
$$

Each state transition probability constitutes a transition probability matrix $P=\left(p_{s t}\right)_{(2 M+4) \times(2 M+4)}$.

From the above definition and analysis of the $2 M+4$ state Markov chain, when the average number of packets reached within a packet transmission delay is $\rho=\lambda E\left(S_{\mathrm{D}-\mathrm{MSMR}-\mathrm{CARQ}}\right)<1$, the Markov chain is irreducible, aperiodic, and normally returned and has a unique steadystate distribution. Set the steady-state distribution as 
$\prod^{\prime}=\left(\pi_{0^{\prime}}, \pi_{1^{\prime}}, \ldots, \pi_{(2 M+3)^{\prime}}\right)$, then according to the equilibrium equation $\prod^{\prime} P^{\prime}=\prod^{\prime}$ and the normalization condition $\sum_{i^{\prime}=0^{\prime}}^{\left(2 M+3^{\prime}\right)} \pi_{i^{\prime}}=1$, we can obtain

$$
\pi_{(M+2),}+\pi_{(M+3),}+\cdots+\pi_{(2 M+3),}=\frac{\left(1-p_{0^{\prime} 0^{\prime}}\right)\left[1-(M+1) p_{1^{\prime} 1^{\prime}}\right]}{1-(M+1) p_{1^{\prime} 1^{\prime}}+p_{0^{\prime} 1^{\prime}}+p_{0^{\prime} 2^{\prime}}+\cdots+p_{0^{\prime}(M+1)^{\prime}}}
$$

As soon as the system enters the states $(M+2)^{\prime}$, $(M+3)^{\prime}, \ldots,(2 M+3)^{\prime}$, the source node $S_{i}(i=1,2$, $\ldots, M)$ will send new packets in the next time slot. Therefore, the sum of the time ratios of DTMC in the states $(M+2)^{\prime},(M+3)^{\prime}, \ldots,(2 M+3)^{\prime}$ is $\pi_{(M+2)^{\prime}}+\pi_{(M+3)^{\prime}}+\cdots+\pi_{(2 M+3)^{\prime}}$, which is the throughput of
D-MSMR-CARQ protocol based on the second relay selection method.

Substituting state transition probability of the system into (11), the throughput of D-MSMR-CARQ protocol based on the second relay selection method is

$$
\mathrm{TH}_{\mathrm{D}-\mathrm{MSMR}-\mathrm{CARQ}}^{\prime}=\frac{\left(1-\prod_{i=1}^{M} p_{S_{i} D} p_{S_{i} R_{b}}\right)\left[1-(M+1)\left(p_{R_{b}} p_{R_{b} D}+1-p_{R_{b}}\right)\left(p_{S} \prod_{i=1}^{M} p_{S_{i} D}+1-p_{S}\right)\right]}{\left\{\begin{array}{c}
1-(M+1)\left(p_{R_{b}} p_{R_{b} D}+1-p_{R_{b}}\right)\left(p_{S} \prod_{i=1}^{M} p_{S_{i} D}+1-p_{S}\right)+\sum_{i=1}^{M}\left[\left(1-p_{S_{i} R_{b}}\right) \prod_{i=1}^{M} p_{S_{i} D} \prod_{j=1}^{i-1} p_{S_{j} R_{b}} \prod_{j=i+1}^{M} p_{S_{j} R_{b}}\right] \\
+\prod_{i=1}^{M} p_{S_{i} D}\left[\prod_{i=1}^{2}\left(1-p_{S_{i} R_{b}}\right) \prod_{j=3}^{M} p_{S_{j} R_{b}}+\left(1-p_{S_{1} R_{b}}\right)\left(1-p_{S_{3} R_{b}}\right) p_{S_{2} R_{b}} \prod_{j=4}^{M} p_{S_{j} R_{b}}+\cdots+p_{S_{M} R_{b}} \prod_{i=1}^{M-1}\left(1-p_{S_{i} R_{b}}\right)+\prod_{i=1}^{M}\left(1-p_{S_{i} R_{b}}\right)\right] p_{R_{b} D}
\end{array}\right\}}
$$

\section{Numerical Results}

In this section, we illustrate the average transmission delay and throughput performance of D-MSMR-CARQ protocol by MATLAB simulations. For simplicity, unless specifically explained, we assume that the simulation parameters used in our evaluations are $L=2, \quad p_{S_{i} R_{b_{i}}}=0.4, t_{T}=1.1 L$, $p_{R_{b_{i}} D}=p_{R_{b} D}=0.1, t_{p}=0.1 L, p_{S_{i} R_{b}}=0.5, p_{S_{i} D}=0.6, M=3$, $N \stackrel{b_{i}}{=} 2, p_{R_{b_{i}}}=p_{S_{i}}=0.9$, and $n=0.2$.

Figures 6 and 7 compare the average transmission delay of D-MSMR-CARQ based on two relay selection methods with that of DH-MSMR-CARQ in [28]. In Figure 6, we investigate the effect of the PER from $S_{i}$ to $R_{b_{i}} / R_{b}$ and the number of source nodes on the average transmission delay. We can see that when the number of source nodes goes up from 1 to 10 , the average transmission delay declines sharply and then remains unchanged after the number of source nodes is 5 . Therefore, the number of source nodes is set as 5 to reduce the system complexity and overhead. Figure 7 depicts the effect of the PER from $S_{i}$ to $R_{b_{i}} / R_{b}$ and the packet length $L$ on the average transmission delay. It can be observed that the average transmission delay increases linearly as the packet length $L$ increases. By adjusting the number of source nodes and the packet length $L$, the D-MSMR-CARQ protocol outperforms the DH-MSMR-CARQ protocol on the average transmission delay performance.

Figure 8 reflects the relationship between the PER from $S_{i}$ to $D$ and the average transmission delay under two relay selection schemes when the packet length $L=2,3$. It can be seen that as the average transmission delay increases, so does the PER from $S_{i}$ to $D$. This is because $S_{i}$ needs cooperative transmission through $R_{b} / R_{b}$ when the PER from $S_{i}$ to $D$ is larger, thereby increasing the system transmission delay. We also see that the first relay selection method has a lower average transmission delay than the second relay selection method, and the average transmission delay is nearly double when $L=3$ compared to $L=2$, which is consistent with the simulations in Figures 6 and 7.

Figure 9 represents the effect of the PER from $S_{i}$ to $D$ on the throughput for two delay selection methods. Observed from Figure 9 that the throughput decreases as the PER from $S_{i}$ to $D$ increases, and the first relay selection method has greater throughput than the second relay selection method. To show the impact of the number of relay nodes on the throughput, we suppose the PER from $S_{i}$ to $D$ is equal to 0.1 , 0.2 , and 0.3 , respectively, in Figure 10 . It is obvious from Figure 10 that the throughput of D-MSMR-CARQ protocol based on the first relay selection method decreases as the PER from $R_{b_{i}}$ to $D$ increases. We can see that when the number of relay nodes goes up from 1 to 10 , the throughput increases gradually and then remains unchanged after the number of relay nodes is 4 . Therefore, the number of relay nodes is set as 4 to reduce the system complexity and overhead.

To better explain the superiority of the proposed protocol, Figure 11 compares the throughput of D-MSMR-CARQ based on the first relay selection method with those of MSMR-CARQ proposed in [27] and DHMSMR-CARQ proposed in [28]. For the purpose, we simulate three MSMR-CARQ protocols under the same simulation parameters. Simulation results show that comparing with D-MSMR-CARQ and MSMR-CARQ, the 


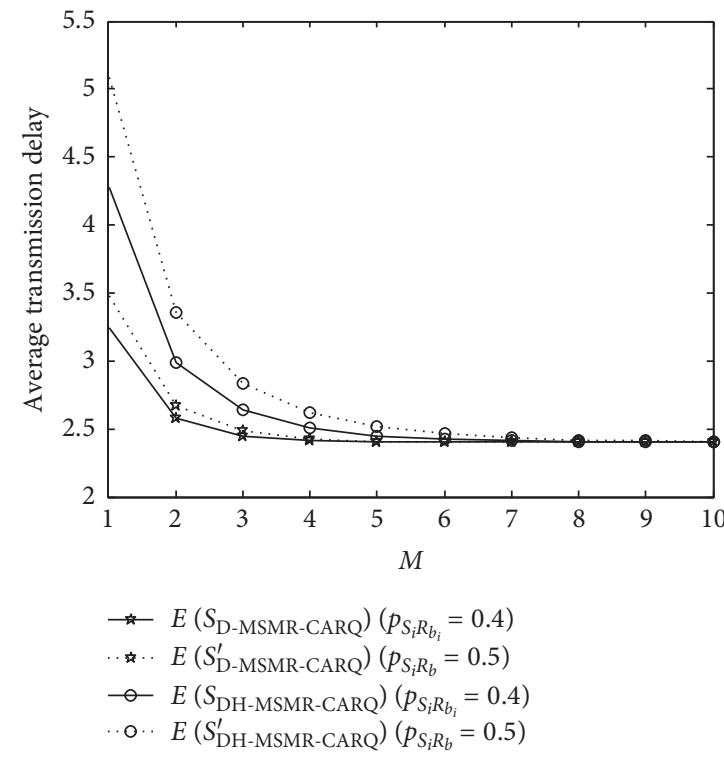

Figure 6: $M$ vs. average transmission delay.

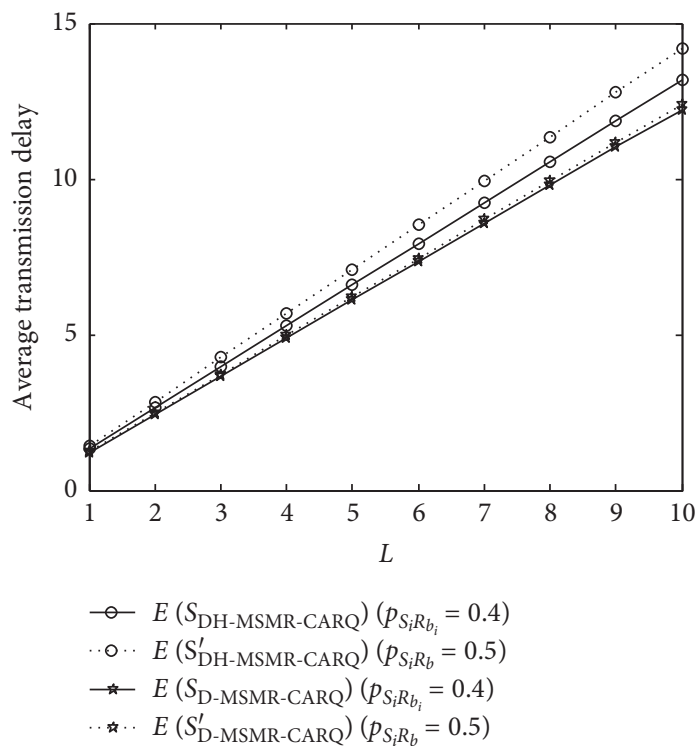

FIgURE 7: $L$ vs. average transmission delay.

throughput of DH-MSMR-CARQ decreases more sharply with the decreasing of the PER from $S_{i}$ to $R_{j}$. The reason is that considering the existence of direct-link from $S_{i}$ to $D$ in a nonharsh channel environment, the throughput performance of the system will be reduced. On the contrary, considering the existence of direct-link will benefit throughput performance in a harsh channel environment. Moreover, when $p_{S_{i} R_{j}}=0.65$, the direct-link has no effect on the throughput which leads to the throughput of D-MSMR-CARQ protocol and DH-MSMRCARQ protocol is equal. As explained above, when $p_{S_{i} R_{j}}=0.95$, the throughput of the MSMR-CARQ protocol and D-MSMR-CARQ protocol is equal; it is also the reason for the intersection of the two curves. 


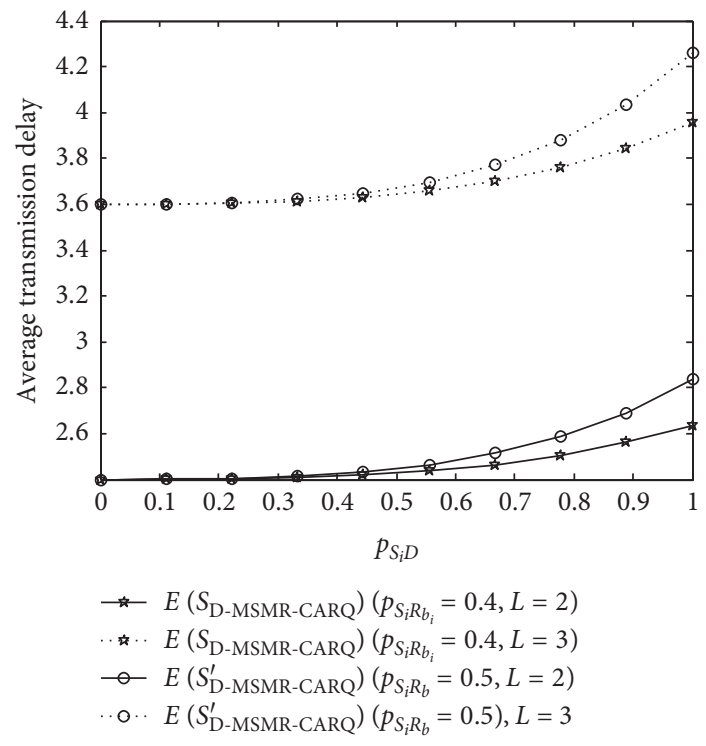

Figure 8: $p_{S_{i} D}$ vs. average transmission delay.

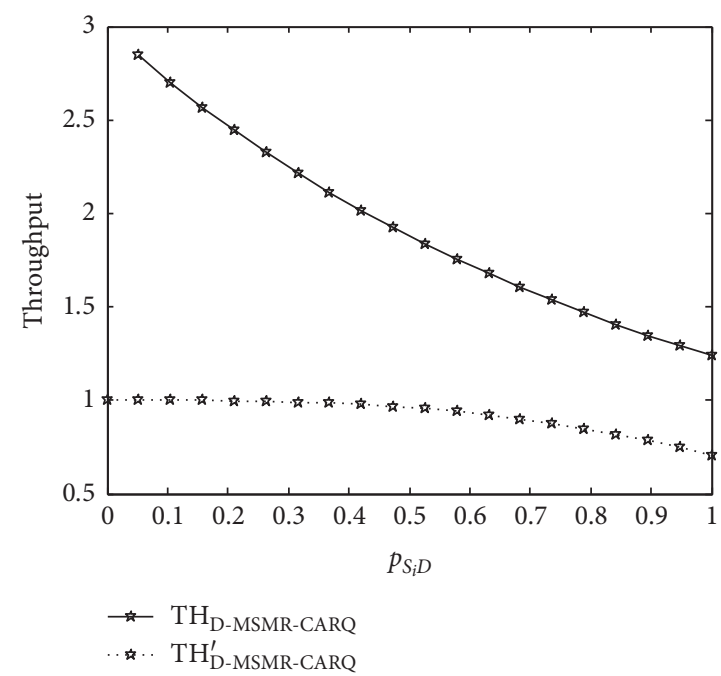

FIGURE 9: $p_{S_{i} D}$ vs. throughput.

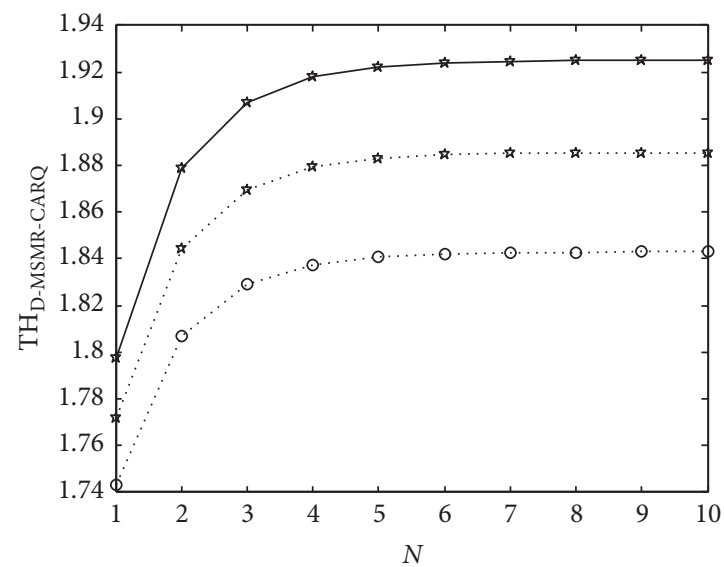

* $\mathrm{TH}_{\text {D-MSMR-CARQ }}\left(p_{R_{b_{i}} D}=0.1\right)$

$\cdots \mathrm{TH}_{\text {D-MSMR-CARQ }}\left(p_{R_{b} D}=0.2\right)$

o.. $\mathrm{TH}_{\text {D-MSMR-CARQ }}\left(p_{R_{b_{i}} D}=0.3\right)$

Figure 10: $N$ vs. throughput. 


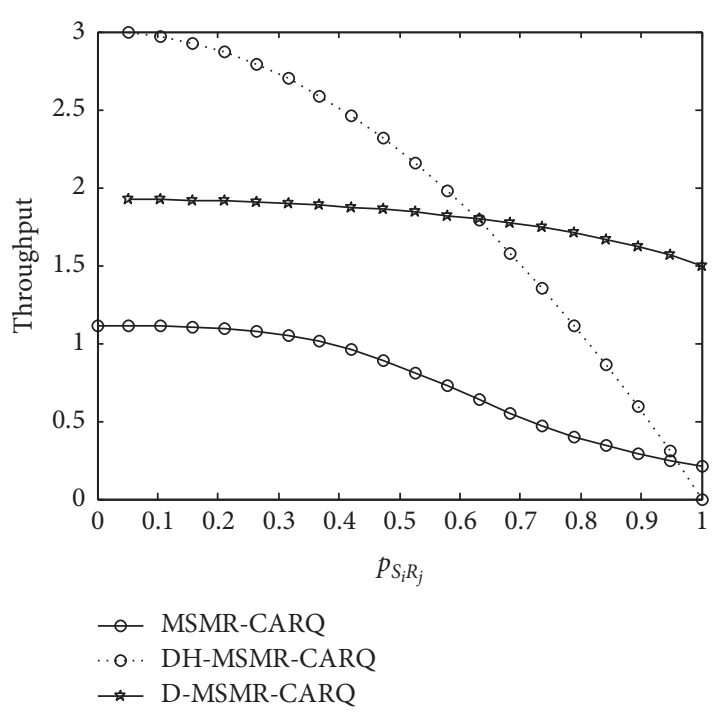

FIgURE 11: Throughput comparison of the three MSMR-CARQ.

\section{Conclusion}

In this paper, we have proposed a D-MSMR-CARQ protocol based on truncation with two relay selection methods. We have investigated the throughput and delay performance by establishing DTMC models for the D-MSMR-CARQ protocol under different relay selection methods. Following this, suppose the packet transmission process and the state space of each DTMC model were obtained. Moreover, for each D-MSMR-CARQ protocol based on different relay selection methods, the closed-form expressions of the average transmission delay were derived by using the average PER and the expressions of the throughput were obtained from the state transition probabilities. Furthermore, we concluded several guidelines for the D-MSMR-CARQ protocol from the numerical simulations: (1) there exists the optimal number of relays and sources deployed in the system when maximizing the throughput and minimizing the transmission delay. (2) For the proposed protocols under two relay selection methods, the protocol based on the first relay selection method has better delay and throughput performance. (3) Direct-link transmission cannot be ignored when the channel environment is harsh. The reason is that for the MSMR-CARQ without direct-link protocol, our proposed protocol improves the delay performance by $13 \%$; moreover, our proposed protocol improves the throughput performance by $47 \%$ when the PER from the source node to the destination node is 0.8 . Finally, the energy consumption of D-MSMR-CARQ protocol based on the first relay selection method can be further investigated in future work.

\section{Data Availability}

The data supporting this article are from previously reported studies and datasets, which have been cited.

\section{Conflicts of Interest}

The authors declare that they have no conflicts of interest.

\section{Acknowledgments}

This project was supported by the National Natural Science Foundation of China (61663024), the PhD Research Startup Foundation of Lanzhou University of Technology (05061405), and the Hongliu Foundation of First-Class Disciplines of Lanzhou University of Technology, China.

\section{References}

[1] I. F. Akyildiz and M. C. Vuran, Wireless Sensor Networks, John Wiley, Hoboken, NY, USA, 2010.

[2] H. Cheng, Z. Su, N. Xiong, and Y. Xiao, "Energy-efficient node scheduling algorithms for wireless sensor networks using Markov Random Field model," Information Sciences, vol. 329, pp. 461-477, 2016.

[3] A. Liu, Z. Chen, and N. N. Xiong, "An adaptive virtual relaying set scheme for loss-and-delay sensitive WSNs," Information Sciences, vol. 424, pp. 118-136, 2018.

[4] D. Banđur, B. Jakšić, M. Banđur, and S. Jović, "An analysis of energy efficiency in wireless sensor networks (WSNs) applied in smart agriculture," Computers and Electronics in Agriculture, vol. 156, pp. 500-507, 2019.

[5] A. Ahmad and L. Zhou, "Patients' adoption of WSN-based smart home healthcare systems: an integrated model of facilitators and barriers," IEEE Transactions on Professional Communications, vol. 60, no. 1, pp. 4-23, 2017.

[6] F. Khan, A. U Rehman, and M. A. Jan, "A secured and reliable communication scheme in cognitive hybrid ARQ-aided smart city," Computers \& Electrical Engineering, vol. 81, Article ID 106502, 2020.

[7] S. Kurt, H. U. Yildiz, M. Yigit, B. Tavli, and V. C. Gungor, "Packet size optimization in wireless sensor networks for smart grid applications," IEEE Transactions on Industrial Electronics, vol. 64, no. 3, pp. 2392-2401, 2017.

[8] M. Yigit, P. Sarisaray Boluk, and V. C. Gungor, "A new efficient error control algorithm for wireless sensor networks in smart grid," Computer Standards \& Interfaces, vol. 63, pp. 27-42, 2019.

[9] B. Sarvi, H. R. Rabiee, and K. Mizanian, "An adaptive crosslayer error control protocol for wireless multimedia sensor networks," Adhoc Networks, vol. 56, pp. 173-185, 2017.

[10] S. Li, Z. Dou, F. Wang, and Q. Xu, "Energy efficiency of five broadcast-based ARQ protocols in multi-hop wireless sensor networks," IET Communications, vol. 13, no. 15, pp. 2243-2253, 2019.

[11] N. Tekin and V. C. Gungor, "The impact of error control schemes on lifetime of energy harvesting wireless sensor networks in industrial environments," Computer Standards \& Interfaces, vol. 70, Article ID 103417, 2020.

[12] I. Dey, M. Majid Butt, and N. Marchetti, "Throughput analysis for virtual MIMO WSNs over measured MIMO channels," IEEE Transactions on Instrumentation and Measurement, vol. 68, no. 1, pp. 297-299, 2019.

[13] X. Zhang, H. He, G. Ankang, and H. Wang, "Analysis of the security performance of multi group multicast centralized large-scale MIMO system," Telecommunication Technology, vol. 60, no. 6, pp. 666-672, 2020, in Chinese.

[14] S. Asheer and S. Kumar, "A comprehensive review of cooperative MIMO WSN: its challenges and the emerging technologies," Wireless Networks, vol. 27, no. 2, pp. 1129-1152, 2021.

[15] S. Asheer and S. Kumar, "Lifetime e through duty cycle in an aggregation based cooperative MIMO framework," Wireless 
Personal Communications, vol. 112, no. 3, pp. 1783-1808, 2020.

[16] H. Wang, G. Yang, Y. Gu, J. Xu, and Z. Sun, "A novel cooperative ARQ method for wireless sensor networks," International Journal of Distributed Sensor Networks, vol. 2015, no. 2, pp. 1-7, 2015.

[17] C.-K. Tseng and S.-H. Wu, "Effective protocols and channel quality control mechanisms for cooperative ARQ with opportunistic AF relaying," IEEE Transactions on Vehicular Technology, vol. 67, no. 3, pp. 2382-2397, 2018.

[18] M. Torabi, W. Ajib, and D. Haccoun, "Performance analysis of amplify and forward cooperative networks with relay selection over Rayleigh fading channels," in Proceedings of the VTC Spring 2009-IEEE 69th Vehicular Technology Conference, pp. 1-5, Barcelona, Spain, April 2009.

[19] R. Arro-Valles, A. Simonetto, and G. Leus, "Consistent sensor, relay, and link selection in wireless sensor networks," Signal Processing, vol. 140, pp. 32-44, 2017.

[20] N. Bissias, G. P. Efthymoglou, and V. A. Aalo, "Performance analysis of dual-hop relay systems with single relay selection in composite fading channels," International Journal of Electronics and Communications (AË̈), vol. 66, no. 1, pp. 39-44, 2012.

[21] D. Zou, D. Deng, Y. Rao, X. Li, and K. Yu, "Relay selection for cooperative NOMA system over correlated fading channel," Physical Communication, vol. 35, Article ID 100702, 2019.

[22] A. A. Rahman, M. N. M. Kahar, and W. I. S. W. Din, "Distance based thresholds for 2-tier relay nodes selection in WSN," Computer Standards \& Interfaces, vol. 66, Article ID 103359, 2019.

[23] Y. Zhou, S. Li, and S. Hou, "Performance analysis of multiple source cooperative ARQ system in wireless sensor networks," Journal of Systems Engineering, vol. 31, no. 5, pp. 584-589, 2016, in Chinese.

[24] R. Alhamad and H. Boujemaa, "Throughput analysis and optimization of relay selection techniques for $\mathrm{m}$ wave communications," Journal of Computer Networks and Communications, vol. 2019, Article ID 7523181, 8 pages, 2019.

[25] L. Yee, S. Mehmood, A. Almogren, I. Ali, and M. H. Anisi, "Improving the performance of opportunistic routing using min-max range and optimum energy level for relay node selection in wireless sensor networks," PeerJ Computer Science, vol. 6, pp. 1-25, 2020.

[26] S. Li, Y. Zhou, D. Peng, Z. Dou, and Y. Zhou, "Analysis of dual-hop and multiple relays cooperative truncated ARQ with relay selection in WSNs," Acta Informatica, vol. 53, no. 1, pp. 1-22, 2016.

[27] Y. Zhou, S. Li, and S. Hou, "Throughput and energy efficiency of multiple sources multiple relays cooperative ARQ system for WSNs," Journal of Computational Information Systems, vol. 10, no. 16, pp. 7085-7092, 2014.

[28] Y. Zhou, D. Lei, B. Wang, and S. Li, "Delay and throughput analysis of DH-MSMR-CATQ based on relay selection in WSNs," Application Research of Computers, vol. 37, no. 12, pp. 3764-3767, 2020, in Chinese. 